\title{
Ação do laser infravermelho em suspensões de Streptococcus mutans
}

\section{Infrared laser action on Streptococcus mutans suspensions}

\author{
Smyrna Luiza Ximenes de Souza ${ }^{1}$ (D) , Stéffani Vasconcelos Santos ${ }^{2}$ (D), Fernanda Freitas Sousa ${ }^{2}$ (D), Ana Larissa Ximenes Batista ${ }^{2}$ (D), \\ Laís Aragão Lima² (D), Ramille Araújo Lima ${ }^{3}$ (D)
}

1. Mestranda em Ciências Odontológicas, Centro Universitário Christus (UNICHRISTUS), Fortaleza, CE, Brasil. 2. Graduanda em Odontologia, Centro Universitário Christus (UNICHRISTUS), Fortaleza, CE, Brasil. 3. Docente do curso de Odontologia, Centro Universitário Christus (UNICHRISTUS), Fortaleza, CE, Brasil.

\section{Resumo}

Introdução: os benefícios do laser infravermelho no tratamento da mucosite oral e no controle da dor na disfunção da articulação temporomandibular são bem reconhecidos e já aplicados clinicamente. Porém, o efeito dessa terapia em bactérias da cavidade oral ainda é incerto. Objetivo: analisar o efeito do laser infravermelho Diodo de Arseneto de Gálio Alumínio (AsGaAl) (LIV) nos níveis de suspensões de Streptococcus mutans UA159. Método: o laser de baixa potência infravermelho e comprimento de onda predominante de 880nm (Therapy XT-ESTM, DMC Equipamentos) foi utilizado com potência de $100 \mathrm{~mW}$ e diâmetro da ponteira $0,0280 \mathrm{~cm}^{2}$. A suspensão bacteriana foi ativada em estufa bacteriológica a $37^{\circ} \mathrm{C}$ e $5 \% \mathrm{CO} 2$, durante um período de 18 horas, ajustada para a concentração de 1-2 x 108 unidades formadoras de colônia/mL (UFC/mL). As suspensões foram submetidas aos seguintes protocolos experimentais e controle: grupo 1) LIV 30s; grupo 2) LIV 60s; grupo 3) LIV 90s; grupo 4) NaCl 0,9\%. Em seguida, uma alíquota foi retirada para a diluição seriada e semeadura das amostras. As placas permaneceram em estufa bacteriológica a $37{ }^{\circ} \mathrm{C}$ e $5 \% \mathrm{CO} 2$ durante um período de 48 horas para posterior contagem das UFC/mL. 0 estudo foi conduzido em triplicata. Resultados: os resultados, a seguir, estão expressos em Log UFC/mL: grupo 1 (8,42 $\pm 0,16)$; grupo $2(8,41 \pm 0,03)$; grupo $3(8,37 \pm 0,11)$; grupo $4(8,37 \pm 0,16)$. Não foi observada diferença estatisticamente entre os grupos (p>0,05). Conclusão: o estudo mostrou que o laser infravermelho, nas condições experimentais usadas, não demonstrou ação na viabilidade de $S$. mutans presentes em suspensões in vitro.

Palavras-chave: Streptococcus mutans. Cárie dentária. Terapia a laser de baixa intensidade. Laser. Odontologia.

\begin{abstract}
Introduction: The benefits of infrared laser in the treatment of oral mucositis and pain control in temporomandibular joint dysfunction are well recognized and applied clinically. However, the effect of this therapy on oral cavity bacteria is still uncertain. Objective: Analyze the effect of infrared laser Gallium Arsenide Diode (AsGaAl) (LIV) on the levels of Streptococcus mutans UA159 suspensions. Method: Low-power infrared laser with predominant wavelength of $880 \mathrm{~nm}$ (Therapy XT-ESTM, DMC Equipment) was used with $100 \mathrm{~mW}$ output power and tip diameter $0.0280 \mathrm{~cm}^{2}$.The bacterial suspension was activated in a bacteriological oven at $37^{\circ} \mathrm{C}$ and $5 \% \mathrm{CO} 2$ for a period of $18 \mathrm{~h}$, adjusted to the concentration of $1-2 \times 108$ colony forming units / mL (CFU / mL).The suspensions were submitted to the following experimental protocols and control: Group 1)LIV 30s; group 2)LIV 60s; Group 3)LIV 90s; Group 4)saline solution. After that, an aliquot was taken for serial dilution and plating of the sample. The plates were kept in a bacteriological oven at $37^{\circ} \mathrm{C}$ and $5 \% \mathrm{CO} 2$ for 48 hours for subsequent cfu/ml counts. The study was conducted in triplicates. Results: The following results are expressed in Log ufc/mL: Group 1 (8.42 \pm 0.16$)$; Group 2 (8.41 \pm 0.03$)$; Group 3 (8.37 \pm 0.11$)$; Group 4 (8.37 \pm 0.16$)$. There was no statistically significant difference between groups (p>0.05). Conclusion: In the experimental tested conditions, the study showed that the infrared laser had no action on the viability of $S$. mutans suspensions in vitro.
\end{abstract}

Key words: Streptococcus mutans. Dental Caries. Low-Level Light Therapy. Lasers. Dentistry.

\section{INTRODUÇÃO}

A laserterapia de baixa intensidade (LBI) refere-se à aplicação da luz (geralmente entregue por meio de um laser de baixa potência diodo ou LED) para promover a reparação dos tecidos, reduzir a inflamação ou induzir analgesia ${ }^{1}$.

As atividades biológicas da LBI baseiam-se em um mecanismo de ação complexo, que pode ser explicado pela absorção de luz dentro das mitocôndrias ${ }^{2}$. A enzima citocromo $c$ oxidase absorve luz vermelha e infravermelha, e essa transferência de luz, na forma de energia irá acarretar a liberação de óxido nítrico $(\mathrm{ON})$ do centro catalítico do citocromo c oxidase (reação redox), formação de oxigênio singleto e aumento subsequente da produção do ânion superóxido (geração de espécies reativas de oxigênio-ROS) ${ }^{3}$. Após a estimulação dos fotorreceptores, ocorre uma cascata de reações bioquímicas na célula que irão promover à liberação de fatores de crescimento por macrófagos, proliferação de queratinócitos, aumento da população e de granulação de mastócitos e angiogênese. Esses efeitos podem levar a uma aceleração no processo de cicatrização de feridas devido, em parte, à redução na duração da inflamação aguda, resultando em uma reparação mais rápida ${ }^{4}$.

Vale salientar as diferenças entre a laserterapia de baixa intensidade das outras modalidades terapêuticas à base de

Correspondente: Ramille Araújo Lima. Unichristus Campus Parque Ecológico. Rua: João Adolfo Gurgel, 133 - Cocó, Fortaleza - CE, 60190-060. E-mail: ramillelima@yahoo.com.br

Conflito de interesse: Não há conflito de interesse por parte de qualquer um dos autores.

Recebido: 19 Set 2018; Revisado: 5 Mar 2019; 20 Mar 2019; Aceito: 27 Mar 2019 
luz. A laserterapia de alta potência promove sua ação por meio da ablação ou do aquecimento de tecidos ou superfícies (como o esmalte dental). A terapia fotodinâmica utiliza a luz, indiretamente, para desencadear reações de fotoativação em fotossensibilizadores (corantes), que, assim, irão gerar radicais livres bactericidas. Em contraste, a LBI usa a ação de luz (e somente luz) para estimular diretamente as células hospedeiras, a fim de reduzir inflamação, aliviar a dor e/ou promover cicatrização ${ }^{1}$.

Os dispositivos de laser ou LED aplicados na LBI, geralmente, emitem luz com comprimentos de onda de 600 a $1000 \mathrm{~nm}$ (vermelho a infravermelho) ${ }^{1}$. Especificamente sobre a luz na região infravermelha (IV) do espectro (acima de $700 \mathrm{~nm}$ ), objeto de estudo da presente pesquisa, sabe-se que esse tipo de luz vem sendo utilizado em tratamentos fisioterápicos há mais de 40 anos. Apesar de seu uso mais recente em odontologia, várias utilizações vêm sendo propostas, tais como:

- redução da dor em pacientes com disfunção temporomandibular ${ }^{5}$;

- cicatrização de feridas ${ }^{6}$;

- tratamento de desordens crônico-inflamatórias, tais como líquen plano oral";

- tratamento de desordens neurossensoriais e dor pósoperatória após cirurgia ortognática ${ }^{8}$;

- redução de dor durante tratamento ortodôntico9;

- redução da dor e auxílio na cicatrização da estomatite aftosa recorrente ${ }^{10}$;

- terapia adjunta no tratamento da osteonecrose dos maxilares ${ }^{11}$;

- terapia adjunta da hipersensibilidade dentinária ${ }^{12}$.

Os efeitos antibacterianos da luz IV em suspensões de Salmonella enterica serovar Typhimurium e Escherichia coli ${ }^{13} \mathrm{e}$ em biofilmes in vivo ${ }^{14}$ e in vitro ${ }^{15,16}$ de Staphylococcus aureus foram estudados por alguns pesquisadores. Por meio de efeitos térmicos e de fotodisrupção, a LBI promove ruptura da parede celular bacteriana e acúmulo de proteína desnaturada no citoplasma bacteriano, levando, assim, a danos letais e subletais à célula ${ }^{17,18}$

Os efeitos da LBI perante os biofilmes de Streptococcus mutans $^{19,20,21}$ e os biofilmes mistos (Streptococcus mutans e Candida albicans $)^{19}$ foram relatados. Apesar da complexidade da comunidade bacteriana que coloniza o biofilme dental, e S. mutans não ser o microrganismo mais numeroso da placa dental, é reconhecido que as glicosiltransferases (Gtfs) de $S$. mutans desempenham papéis críticos no desenvolvimento da virulência da placa dentária. Os genes Gtf, entre outras funções, são responsáveis pela produção da matriz de polissacarídeos solúveis e insolúveis ${ }^{22}$. Além disso, apresentam a habilidade em rapidamente degradar carboidratos fermentáveis, promovendo a formação abundante de ácido e a capacidade de tolerar ambientes com baixo $\mathrm{pH}^{23}$. Em adição, S. mutans tem alta afinidade à superfície dentária mediada pela presença de adesinas e polissacarídeos extracelulares que contribuem para a sua patogenicidade ${ }^{24}$.
Atualmente, encontra-se estabelecido na comunidade científica o conceito de que o surgimento das duas doenças mais prevalentes na cavidade bucal de humanos, cárie dental e doença periodontal estejam intimamente relacionadas à presença de biofilme. O tratamento das doenças relacionadas à presença dos biofilmes bacterianos envolve, basicamente, a remoção mecânica desses biofilmes e o uso de antibióticos e agentes antissépticos. Entretanto, diante da emergência de cepas resistentes aos agentes antimicrobianos tradicionais, tem aumentado o interesse da comunidade científica em desenvolver terapias antimicrobianas alternativas que tornem o surgimento improvável de cepas geneticamente resistentes ${ }^{25}$.

Os benefícios do laser infravermelho no tratamento de diversas patologias da cavidade oral (redução de dor e cicatrização) são bem reconhecidos e já aplicados clinicamente. Porém, o efeito dessa terapia em bactérias da cavidade oral ainda é incerto. Portanto, o objetivo deste trabalho é analisar o efeito antimicrobiano do laser infravermelho de Arseneto de Gálio Alumínio (AsGaAl) em suspensões de S. mutans UA159, vislumbrando um método alternativo de controle de biofilmes cariogênicos.

\section{MATERIAIS E MÉTODOS}

\section{Fonte de Luz}

A fonte de luz utilizada foi um Laser Infravermelho Diodo de Arseneto de Gálio Alumínio (AsGaAl) com comprimento de onda predominante de $880 \mathrm{~nm}$. A potência de luz de Laser foi $100 \mathrm{mw}$ e o diâmetro da ponteira é $0.028 \mathrm{~cm}^{2}$ (Therapy XTESTM, DMC Equipamentos, São Carlos, SP, Brasil).

O local irradiado foi o poço de uma placa de cultura de células de 96 poços, cuja área é $0,321 \mathrm{~cm}^{2}$. Três tempos de irradiação foram utilizados: 30 segundos, 60 segundos e 90 segundos. Assim, foram utilizadas as seguintes doses de energia $e$ respectivas densidades de energia: 1) $3 \mathrm{~J}$ e 9,34 J/cm²; 2) $6 \mathrm{~J} \mathrm{e}$ $18,69 \mathrm{~J} / \mathrm{cm}^{2}$; 3) $9 \mathrm{~J}$ e $28,03 \mathrm{~J} / \mathrm{cm}^{2}$. A distância entre a fonte de luz e o local da irradiação foi de $1 \mathrm{~mm}$.

\section{Preparo do inóculo}

O microrganismo utilizado foi o S. mutans UA159 cujo inóculo foi preparado em Tryptone Soya Broth (TSB), contendo 1\% de glicose em temperatura de $37^{\circ} \mathrm{C}$ e atmosfera parcial de $5 \%$ $\mathrm{CO}^{2}$. A suspensão permaneceu em estufa por um período de 18 horas e, após esse período, foi ajustada por meio de espectrofotômetro, a fim de se obter uma suspensão com 108 unidades formadoras de colônia (UFC) por $\mathrm{mL}$ de suspensão.

\section{Delineamento experimental}

O experimento apresentou os seguintes grupos:

- grupo 1: $100 \mu \mathrm{L}$ de caldo $+100 \mu \mathrm{L}$ do inóculo + LUZ por 30 segundos. 
- grupo 2: $100 \mu \mathrm{L}$ de caldo + $100 \mu \mathrm{L}$ do inóculo + LUZ por 60 segundos.

- grupo 3: $100 \mu \mathrm{L}$ de caldo + $100 \mu \mathrm{L}$ do inóculo + LUZ por 90 segundos.

- grupo 4: $200 \mu \mathrm{L}$ de solução salina ( $\mathrm{NaCl}$ 0,89\%).

Imediatamente após a aplicação do tratamento, de acordo com o grupo experimental, o volume total de cada poço (200 $\mu \mathrm{L}$ ) foi transferido para tubos de microcentrífuga de $1,5 \mathrm{~mL}$ e, em seguida, diluições decimais seriadas $\left(10^{-1}\right.$ a $\left.10^{-7}\right)$ foram realizadas. $O$ material foi semeado em placas de petri, contendo meio de cultura BHI ágar e incubado em condições ideais para o crescimento desses microrganismos (estufa de $\mathrm{CO} 2$ a $5 \%$, a $37^{\circ} \mathrm{C}$ por 48 horas). Após esse período, as colônias foram contadas com a ajuda de um contador de colônias, e a relação de unidades formadoras de colônia por mililitro (UFC/mL) foi estabelecida.

\section{Análise dos dados}

Os resultados foram transformados para logaritmo na base dez e foram calculados as médias e os desvios-padrão de cada grupo. Os dados foram submetidos ao teste de normalidade de Kolmogorov-Smirnov, e foi realizado o teste ANOVA seguido de Tukey. Foi estabelecido um intervalo de confiança de $95 \%$ e nível de significância $5 \%$. O programa estatístico utilizado foi o GraphPrism 5.0.

\section{RESULTADOS}

Os resultados, a seguir, estão expressos em Log UFC/mL, em que são demonstrados valores médios \pm desvio-padrão para o grupo $1(8,42 \pm 0,16)$; grupo $2(8,41 \pm 0,03)$; grupo $3(8,37 \pm 0,11)$ e grupo $4(8,37 \pm 0,16)$. Não foi observada diferença, estatisticamente, entre os grupos $(p>0,05)$ (figura 1$)$.

Figura 1. Gráfico da análise da viabilidade bacteriana (log CFU/ $\mathrm{mL}$ ) entre os grupos experimentais testados.

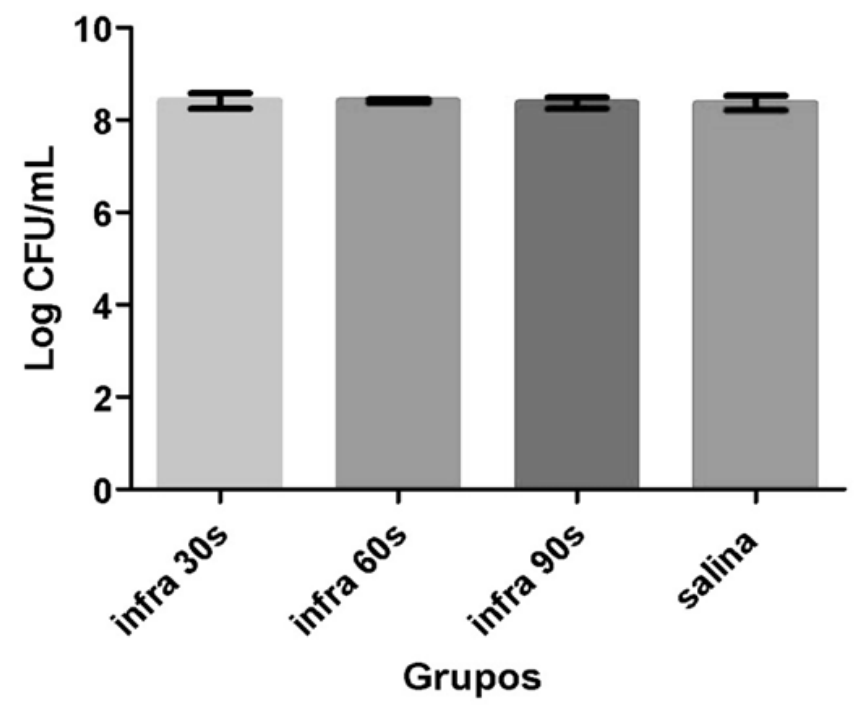

\section{DISCUSSÃO}

Apesar da redução de sua incidência, a cárie é uma das doenças crônicas mais prevalentes do mundo ${ }^{26}$, atingindo cerca de $60 \%$ a $90 \%$ das crianças em idade escolar e quase $100 \%$ da população adulta ${ }^{27}$. É evidente, portanto, a necessidade de mais estudos sobre alternativas de prevenção e controle do biofilme cariogênico.

Basso et al. (2011) 19 observaram redução de 1-log CFU/mL em biofilmes de $S$. mutans com a aplicação do laser InGaAsP (780 $\mathrm{nm}$ ). Neste estudo, foi utilizado um laser com potência de 0,4 $\mathrm{W}$ por $1000 \mathrm{~s}$ (dose de energia $=400 \mathrm{~J}$ ) para alcançar a redução citada. Os mesmos pesquisadores testaram as doses de $100 \mathrm{~J}$ e $200 \mathrm{~J}$ e não observaram redução na viabilidade bacteriana, assim como demonstrado na presente pesquisa. Ressalta-se que, neste estudo, foi utilizada uma dose de energia máxima de $9 \mathrm{~J}$, dose 44 vezes menor do que a dose relativa à pesquisa citada anteriormente.

Os efeitos da luz azul, com comprimento de onda de 400$500 \mathrm{~nm}$, sobre biofilmes de S. mutans foi estudado por Chebath-Taube e colaboradores (2012)20. Embora tal luz seja, geralmente, utilizada para fotopolimerização de compósitos de resina, ela tem sido estudada como uma possível fonte de ação contra infecções por microrganismos. Os autores observaram um decréscimo na contagem unidades formadoras de colônia no grupo exposto à luz azul por sete $\left(476 \mathrm{~J} / \mathrm{cm}^{2}\right)$ ou dez minutos $\left(680 \mathrm{~J} / \mathrm{cm}^{2}\right) \mathrm{em}$ um biofilme de seis horas.

Sousa et al $(2015)^{21}$ pesquisaram o uso da luz azul, com comprimento de onda de $420 \mathrm{~nm}$, para prevenir o desenvolvimento da matriz de polissacarídeos em biofilme de S. mutans com aplicação duas vezes ao dia, ao longo de cinco dias. Utilizaram uma exposição de 12,56 minutos, com uma densidade de energia de $72 \mathrm{~J} / \mathrm{cm}^{2}$. Perceberam que a exposição à luz azul reduziu a concentração de polissacarídeo extracelular insolúvel comparado aos outros grupos28, o que pode acarretar a diminuição da cariogenicidade do biofilme sem a redução da quantidade de microrganismos. De acordo com Dai et al $(2012)^{28}$, a luz azul pode induzir respostas fisiológicas mediadas pelos receptores de luz azul; dessa forma, esta pode regular a motilidade bacteriana, suprimir o desenvolvimento do biofilme e potenciar a inativação da luz das bactérias.

Rocha et al. (2014) 29 $^{29}$ estudaram os efeitos da irradiação com laser de baixa intensidade de Arsenato de Gálio sobre cepas de Candida albicans, Candida krusei e Candida tropicalis, em doses de $1,2 \mathrm{~J} / \mathrm{cm}^{2}$ (10 segundos), $3,7 \mathrm{~J} / \mathrm{cm}^{2}$ (30 segundos), $7,5 \mathrm{~J} / \mathrm{cm}^{2}$ (um minuto) e $15 \mathrm{~J} / \mathrm{cm}^{2}$ (dois minutos). Seus resultados mostraram diferença significativa estatisticamente de acordo com tais doses aplicadas, o que discorda dos presentes resultados obtidos com S. mutans, a laserterapia de baixa intensidade, demonstrando o estudo anterior efeito inibitório sobre as cepas de Candida citadas, sugerindo maior sensibilidade de fungos à terapia com laser. Do mesmo modo Zhang et al (2016) $)^{30}$ estudaram a luz azul e seu efeito para inativação da Candida albicans in vitro e 
em queimaduras infectadas de ratos. Apesar de não ter havido diferença estatística significativa, o estudo demonstrou que a C. albicans foi susceptivel à luz azul e mostrou que uma única exposição a essa luz $\left(432 \mathrm{~J} / \mathrm{cm}^{2}\right)$ a reduziu, significativamente, nas queimaduras.

Pereira e colaboradores (2014) ${ }^{14}$ compararam os efeitos da laserterapia de baixa intensidade no crescimento in vitro de Staphylococcus aureus em feridas infectadas in vivo, utilizando laser Arsenato de Gálio com 904 nm de comprimento de onda. Assim como no presente estudo, os autores não observaram redução da viabilidade bacteriana, após uso da laserterapia. Analisando in vitro biofilmes de $S$. aureus meticilina-resistentes, Krespi et al (2011) $)^{15}$ testaram o efeito do laser IV (940 nm, 3 W, 180 segundos) e também não observaram redução da viabilidade bacteriana. Vale ressaltar que os autores demonstraram uma redução significativa quando o uso laser IV foi realizado em combinação com o laser de comprimento de onda de $300 \mathrm{~nm}$. De forma semelhante, Tran et al (2018) ${ }^{16}$ observaram uma redução da viabilidade bacteriana em biofilmes de $S$. aureus quando a combinação laser IV (780 nm - $1060 \mathrm{~nm}$ ) e laser azul (405 nm) foi utilizada, porém não observaram essa redução quando o laser IV foi utilizado sozinho. Esses resultados corroboram os encontrados na presente pesquisa, em que não observamos efeito antimicrobiano do laser IV.

\section{CONCLUSÃO}

O presente estudo mostrou que o laser infravermelho AsGaAl $(\lambda=880 \mathrm{~nm})$ não demonstrou ação na viabilidade bacteriana de suspensões in vitro de $S$. mutans. Mais estudos são necessários com o intuito de observar os efeitos do laser infravermelho em diferentes densidades de energia e em combinação com outras fontes de luz em diferentes comprimentos de onda.

\section{AGRADECIMENTOS}

Os autores agradecem ao Centro Universitário Christus pela concessão de bolsa de iniciação científica aos autores SVS, FFS, ALXB.

Os autores agradecem ao Conselho Nacional de Desenvolvimento Científico e Tecnológico - CNPq pela concessão de bolsa de iniciação científica ao autor LAL

\section{REFERÊNCIAS}

1. Carroll JD, Milward MR, Cooper PR, Hadis M, Palin WM. Developments in low level light therapy (LLLT) for dentistry. Dent Mater. 2014 May; 30(5):465-475. doi: 10.1016/j.dental.2014.02.006.

2. Karu TI. Mitochondrial mechanisms of photobiomodulation in context of new data about multiple roles of ATP. Photomed Laser Surg. 2010 Apr; 28(2): 159-60. doi: 10.1089/pho.2010.2789.

3. Karu T, Pyatibrat L, Kalendo G. Photobiological modulation of cell attachment via cytochrome c oxidase. Photochem Photobiol Sci. 2004 Feb; 3(2): 211-6. Doi: 10.1039/b306126d

4. Lim W, Kim J, Kim S, Karna S, Won J, Jeon SM, Kim SY, Choi Y, Choi H, Kim O. Modulation of lipopolysaccharide-induced NF-kappa-B signaling pathway by $635 \mathrm{~nm}$ irradiation via heat shock protein 27 in human gingival fibroblast cells. Photochem Photobiol. 2013 Jan-Feb; 89(1): 199-207. doi: 10.1111/j.17511097.2012.01225.x.

5. Panhoca VH, De Fatima Z, Lizarelli R, Nunez SC, Pizzo RC, Grecco C, Paolillo FR, Bagnato VS. Comparative clinical study of light analgesic effect on temporomandibular disorder (TMD) using red and infrared led therapy. Lasers Med Sci. 2015 Feb; 30(2): 815-822. doi: 10.1007/s10103-013-1444-9.

6. Damante CA, Marques MM, Micheli GD. Terapia com laser em baixa intensidade na cicatrização de feridas - revisão de literatura. RFO. 2008; $13(3): 88-93$

7. Al-Maweri SA, Kalakonda B, Al-soneidar WA, Al-Shamiri HM, Alakhali MS Alaizari N. Efficacy of low-level laser therapy in management of symptomatic oral lichen planus: a systematic review. Lasers Med Sci. 2017; 32(6), 1429-1437. doi: 10.1007/s10103-017-2233-7.

8. Pol R, Gallesio G, Riso M, Ruggiero T, Scarano A, Mortellaro C, et al. Effects of Superpulsed, Low-Level Laser Therapy on Neurosensory Recovery of the Inferior Alveolar Nerve. J Craniofac Surg. 2016;27(5):1215-9.

9. Deana NF, Zaror C, Sandoval P, Alves N. Effectiveness of Low-Level Laser Therapy in Reducing Orthodontic Pain: A Systematic Review and Meta-Analysis. Pain Res Manag. 2017 Sep; 2017: 1-18. doi: https://doi.org/10.1155/2017/8560652
10. Suter VGA, SJölund S, Bornstein MM. Effect of laser on pain relief and wound healing of recurrent aphthous stomatitis: a systematic review. Lasers Med Sci. 2017 May; 32(4): 953-963. doi: 10.1007/s10103-017-2184-z.

11. Vescovi P, Manfredi M, Merigo E, Guidotti R, Meleti M, Pedrazzi G, et al. Early surgical laser-assisted management of bisphosphonate-related osteonecrosis of the jaws (BRONJ): a retrospective analysis of 101 treated sites with longterm follow-up. Photomed Laser Surg. 2012 Jan; 30(1): 5-13. doi: 10.1089/ pho.2010.2955

12. Ladalardo TCCGP, Pinheiro A, Campos RAC, Brugnera A Júnior, Zanin F, Albernaz PLM, et al. Laser Therapy in the Treatment of Dentine Hypersensitivity. Braz Dent J. 2004;15(2): 144-150. doi: http://dx.doi.org/10.1590/S010364402004000200011

13. Ha JW, Kang DH. Simultaneous near-infrared radiant heating and UV radiation for inactivating Escherichia coli 0157:H7 and Salmonella enterica serovar Typhimurium in powdered red pepper (Capsicum annuum L.). Appl Environ Microbiol. 2013 Nov; 79(21): 6568-75. doi: 10.1128/AEM.02249-13.

14. Pereira PR, Paula JB, Cielinski J, Pilonetto M, Von Bahtem, LC. Effects of low intensity laser in in vitro bacterial culture and in vivo infected wounds. Rev. Col. Bras. Cir. 2014 Jan-Feb; 41(1): 49-55.

15. Krespi YP, Kizhner V, Nistico L, Hall-Stoodley L, Stoodley P. Laser disruption and killing of methicillin-resistant Staphylococcus aureus biofilms. Am J Otolaryngol. 2011 May-Jun; 32(3):198-202. doi: 10.1016/j.amjoto.2010.01.010.

16. Tran VN, Dasagrandhi C, Truong VG, Kim YM, Kang HW. Antibacterial activity of Staphylococcus aureus biofilm under combined exposure of glutaraldehyde, near-infrared light, and 405-nm laser. PLoS One. 2018 Aug; 13(8):e0202821. doi: 10.1371/journal.pone.0202821.

17. Dworkin M. Endogenous photosensitization in a carotinoidless mutant of Rhodopseudomonas speroides. J Gen Physiol .1958 Aug; 41(16):1099-1112. doi: $10.1085 /$ jgp.41.6.1099.

18. Rosenberg B, Kemeny G, Switzer RC, Hamilton TC. Quantitative evidence for protein denaturation as the cause of thermal death. Nature 1971; 232: 471-473. 
19. Basso FG, Oliveira CF, Fontana A, Kurachi C, Bagnato VS, Spolidório DM, et al. In vitro effect of low-level laser therapy on typical oral microbial biofilms. Braz Dent J. 2011; 22(6): 502-10.

20. Chebath-Taub D, Steinberg D, Featherstone JDB, Feuerstein O. Influence of blue light on Streptococcus mutans re-organization in biofilm. J Photochem Photobiol B. 2012 Nov; 116:75-78. doi: 10.1016/j.jphotobiol.2012.08.004

21. Sousa DL, Lima RA, Zanin IC, Klein ML, Janal MN, Duarte S. Effect of twicedaily blue light treatment on matrix-rich biofilm development. PLos ONE. 2015; 10(7): e0131941/1-12. doi: 10.1371/journal.pone.0131941.

22. Bowen $\mathrm{WH}$, Koo H. Biology of Streptococcus mutans-derived glucosyltransferases: role in extracellular matrix formation of cariogenic biofilms. Caries Res. 2011; 45(1): 69-86. doi: 10.1159/000324598.

23. Svensater G, Welin J, Wilkins J. Protein expression by planktonic and biofilm cells of Streptococcus mutans. FEMS Microbiology Letters. 2001 Nov; 205(1):139-46.

24. Senadheera MD, Guggenheim B, Spatafora GA, Huang YC, Choi J, Hung DCl, Treglown JD, Goodman SD, Ellen RP, Cvitkovitch DGA. Signal Transduction System in Streptococcus mutans Affects gtfBCD, gbpB, and ftf Expression, Biofilm Formation, and Genetic Competence Development. J Bacteriol. 2005 Jun;187(12):4064-4076.
25. Hamblin MR, Hasan T. Photodynamic therapy: a new antimicrobial approach to infectious desease? Photochem Photobiol Sci. 2004 May; 3(5): 436-450.

26. Marcenes W, Kassebaum NJ, Bernabé E, Flaxman A, Naghavi M, Lopez A, et al. Global Bueden of Oral Conditions in 1990-2010: a systematic analysis. J Dent Res. 2013 May; 92(7):592-597. doi: 10.1177/0022034513490168.

27. Petersen PE, Bourgeois D, Ogawa H, Estupinan-ᄀday S, Ndiaye C. The global burden of oral diseases and risks to oral health. Bull World Healh Organ. 2005 Sep; 83(9): 661-־9. doi: /S0042-96862005000900011.

28. Dai T, Gupta A, Murray CK, Vrahas MS, Tegos GP, Hamblin MR. Blue light for infectious diseases: Propionibacterium acnes, Helicobacter pylori, and beyond? Drug Resist Updat. 2012 Aug; 15(4): 223-236. doi: 10.1016/j.drup.2012.07.001.

29. Rocha EALSS, Cardoso AMR, Limeira FIR, Medeiros ACD, Catao MHCV, Gomes DQC. Low-level laser irradiation on Candida strains: an in vitro study. Rev Cubana Estomatol. 2015 Jan; 51(4): 358-365.

30. Zhang $Y$, Zhu $Y$, Chen J, Wang $Y$, Sherwood ME, Murray MSV, Hooper DC, Hamblin MR, Dai T. Antimicrobial blue light inactivation of Candida albicans: In vitro and in vivo studies. Virulence. 2016 Jul; 7(5): 536-545. doi: 10.1080/21505594.2016.1155015 